\title{
Seroprevalencia de virus varicela-zoster en niños con cáncer en seis hospitales de Santiago, Chile
}

\author{
Giannina Izquierdo, Marcela Zubieta, María J. Martínez G, Ana M. Álvarez, Carmen L. Avilés, \\ Ana Becker, Mónica Peña, Carmen Salgado, Pamela Silva, Santiago Topelberg, Juan Tordecilla, \\ Mónica Varas, Milena Villarroel, Tamara Viviani y María E. Santolaya. Programa Nacional Infantil \\ de Drogas Antineoplásicas (PINDA), Subcomité de Enfermedades Infecciosas, Santiago, Chile
}

\section{Seroprevalence of varicella-zoster virus in children with cancer in six hospitals in Santiago, Chile}

Infections with varicella-zoster virus (VVZ) in immunocompromised children imply a high mortality. There is no data about VVZ seroprevalence in children with cancer in our country. Aim: To determine the prevalence of VVZ antibodies in children with cancer who have undergone chemotherapy or have undergone a hematopoietic stem cell transplant. Methodology: collaborative, multicenter study. Serum samples were collected from 281 children with cancer and episodes of febrile neutropenia from 6 hospitals belonging to the public health network in the Metropolitan Region between June 2004 and August 2006. These samples were stored at $-70{ }^{\circ} \mathrm{C}$, and 200 of them were randomly chosen and analyzed to determine VVZ IgG (ELISA). Results: 179 samples from 179 children, 65\% male. Ninety eigth/179 (55\%) were positive, $72 / 179(40 \%)$ negative and 9/179 (5\%) indeterminate. Stratified by age, seropositive percentage was: 1 to 4 years 32\%, 5-9 years 42\%, 10-14 years 78\%, over 15 years $88 \%$. Conclusion: Forty percent of children treated for cancer are seronegative to VVZ infection, a frequency that decreases with age. These results support the adoption of preventive measures to avoid infection in this population of children at risk of developing a serious and possibly fatal illness.

Key words: Seroprevalence, varicella-zoster virus, immunocompromised.

Palabras clave: Seroprevalencia, virus varicela-zoster, inmunocomprometidos.

\section{Introducción}

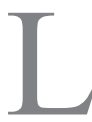

a infección primaria por virus varicela-zoster (VVZ) produce varicela y su reactivación da origen al cuadro de herpes zoster. La varicela es una enfermedad frecuente y altamente transmisible en la infancia, de curso generalmente benigno y autolimitado en hospederos inmunocompetentes ${ }^{1}$.

En niños con cáncer, la infección por VVZ puede producir una enfermedad grave ${ }^{2}$, debido a la disfunción de la inmunidad provocada por la enfermedad de base y/o el tratamiento con fármacos antineoplásicos o corticoesteroidales $^{3-5}$.

Estudios serológicos realizados en E.U.A. han comprobado que $90 \%$ de los adultos están inmunes a $\mathrm{VVZ}^{6}$. En América Latina, se han evidenciado anticuerpos en $85 \%$ de la población en forma global, $57 \%$ en los niños bajo los 5 años, $86 \%$ entre los 6 y 10 años y en $95 \%$ de los adolescentes y adultos ${ }^{7}$.

En Chile los datos son escasos; un estudio no publicado realizado en el sector norte de Santiago, en 503 sujetos sanos de 1 a 39 años de edad, mostró una seroprevalencia de $35,6 \%$ entre 1 y 4 años; $61,7 \%$ entre 5 y 9 años; $90 \%$ entre 10 y 14 años y sobre $95 \%$ en población adulta ${ }^{8}$.
En nuestro país, cada año se diagnostican aproximadamente 500 casos nuevos de cáncer en niños bajo 15 años de edad. Se estima que el Sistema Público de Salud, a través del Programa Nacional de Cáncer Infantil y Drogas Antineoplásicas (PINDA) asume el tratamiento de 80\% de esta población ${ }^{9}$. De estos pacientes, $60 \%$ son tratados en las seis Unidades de Oncología de los hospitales pediátricos de la Región Metropolitana.

Se desconoce la seroprevalencia de VVZ en pacientes oncológicos pediátricos en tratamiento quimioterápico. El objetivo de este trabajo fue determinar la prevalencia de anticuerpos anti VVZ en niños con cáncer sometidos a quimioterapia o trasplante de precursores hematopoyéticos (TPH).

\section{Material y Método}

Diseño global del estudio. Luego de obtener la aprobación del Comité de Ética del Ministerio de Salud de Chile (MINSAL), se tomaron al azar 200 muestras de suero congeladas a $-70^{\circ}$ que habían sido obtenidas entre junio de 2004 y agosto de 2006, como parte de un estudio prospectivo, colaborativo y multicéntrico en que

\author{
Universidad de Chile \\ Facultad de Medicina \\ Programa de Formación de \\ Especialistas en Infectología \\ Pediátrica (GI). \\ Instituto de Ciencias Biomédicas, \\ Programa de Virología (MJM, MP) \\ Hospital Exequiel González \\ Cortés (MZ, CS) \\ Hospital Luis Calvo \\ Mackenna (MV, MES). \\ Hospital San Juan \\ de Dios (AMA, MV). \\ Hospital San Borja \\ Arriarán (CLA, PS) \\ Hospital Sotero del Río (AB, TV). \\ Hospital Roberto del Río (ST, JT).
}

Financiamiento: Fundación Nuestros Hijos y FONDECYT 1040907 Programa Virología, I.C.B.M. Facultad de Medicina, Universidad de Chile.

Los autores declaran que no existen conflictos de intereses.

Recibido: 1 de enero de 2012 Aceptado: 10 de octubre de 2012

Correspondencia a: Marcela Zubieta mzubieta@fnh.cl 
Tabla 1. Distribución de la muestra vs población en tratamiento por cáncer por hospital

\begin{tabular}{|lcccc} 
& \multicolumn{2}{c}{ Muestras estudiadas } & \multicolumn{2}{c}{$\begin{array}{c}\text { Pacientes con cáncer } \\
*\end{array}$} \\
\cline { 3 - 5 } & n pacientes & $\%$ & n pacientes & $\%$ \\
ECG & 31 & 17,3 & 43 & 14,3 \\
LCM & 73 & 40,8 & 116 & 38,6 \\
RR & 40 & 22,3 & 64 & 21,3 \\
SB & 6 & 3,4 & 13 & 4,3 \\
SJ & 15 & 8,4 & 43 & 14,3 \\
S de R & 14 & 7,8 & 21 & 7,0 \\
Total pacientes & 179 & & 300 & $\mathrm{p}=0,69 *$ \\
\hline
\end{tabular}

ECG: Hospital Exequiel González Cortés; LCM: Hospital Luis Calvo Mackenna; RR: Hospital Roberto del Río; SB: Hospital San Borja Arriarán; SJ: Hospital San Juan de Dios; S de R: Hospital Sótero del Río

Figura 1. Seroprevalencia de VVZ en niños con cáncer en tratamiento en 6 hospitales de Santiago, Chile. participaron 281 niños provenientes de seis hospitales de la Región Metropolitana adscritos al PINDA: Luis Calvo Mackenna, Roberto del Río, Exequiel González Cortés, Sótero del Río, San Borja Arriarán y San Juan de Dios. Habían sido criterios de inclusión a dicho estudio el tener menos de 18 años, encontrarse en tratamiento quimioterápico o haber recibido un TPH y estar cursando con un episodio de neutropenia febril (NF). Se excluyeron aquellas muestras que venían con información incompleta o eran episodios repetidos de un mismo paciente. Las muestras se sometieron a determinación de IgG anti VVZ por método ELISA.

Determinación de IgG anti VVZ. Los sueros analizados fueron centrifugados y mantenidos en alícuotas a $-70^{\circ} \mathrm{C}$ en el Laboratorio de Virología I.C.B.M., Facultad de Medicina, Universidad de Chile. Se utilizó el test Ridascreen ${ }^{\circledR}$ VVZ IgG ELISA, (R-Biopharm, Darmstadt, Germany) de acuerdo a las especificaciones del fabricante. Luego de ajustar los reactivos y placas a temperatura ambiente, se agregaron $100 \mu \mathrm{l}$ de cada suero diluido, controles positivos, negativos y blanco a los respectivos pocillos y se incubaron a $37^{\circ} \mathrm{C}$ durante $30 \mathrm{~min}$. Posteriormente se realizaron lavados antes de agregar el conjugado y de incubar en iguales condiciones. Se procedió de manera similar con el sustrato y se finalizó con la solución de detención. Luego se realizó la evaluación fotométrica a 450/620 nm. El nivel de corte se obtuvo de los datos aportados por el fabricante. Las absorbancias bajo $0,15 \mathrm{U} /$ $\mathrm{ml}$ fueron catalogadas como negativas y sobre $0,22 \mathrm{U} / \mathrm{ml}$ fueron positivas. Las muestras que resultaron entre 0,15 y $0,22 \mathrm{U} / \mathrm{ml}$ fueron definidas como indeterminadas y se repitieron en un ensayo diferente. Las muestras que por segunda vez dieron resultados de absorbancia en el rango de indefinido, permanecieron en esa categoría.

Análisis estadístico. Se utilizó test de t-student y $\square^{2}$ para el análisis de los resultados. Se consideró significativo un valor de $\mathrm{p}<0,05$.

\section{Resultados}

Población estudiada. De las 200 muestras de suero obtenidas, se excluyeron 21; doce por pertenecer a un mismo paciente en diferentes episodios de NF y 9 por falta de información, realizándose determinación de IgG anti VVZ en 179 muestras de 179 pacientes. La distribución fue representativa de la población de niños en tratamiento en cada uno de los seis centros en términos porcentuales (p > 0,69) (Tabla 1). El 65\% del grupo analizado fue de género masculino, con una mediana de edad de 8 años (rango: 2 meses a 18 años).

Determinación de $\operatorname{IgG}$ anti $V V Z$. Del total de niños estudiados, $98(55 \%)$ resultó positivo para la determi-

Figura 2. Distribución de serotipos para VVZ según grupo etáreo.

$\square$ Seronegativos

$\square$ Seropositivos

$* p<0,05$ 
nación de anticuerpos anti VVZ, 72 negativo (40\%) y 9 indeterminado (5\%). (Figura 1). Estratificado por grupo etario, el nivel de IgG anti VVZ sobre el valor de corte estuvo presente en : $32 \%$ de los niños entre 1 y 4 años, $42 \%$ entre 5 y 9 años, $78 \%$ entre 10 y 14 años y $88 \%$ de los mayores de 15 años (Figura 2). Los niños bajo los 10 años fueron, en proporción, significativamente menos seropositivos que los mayores de 10 años $(p<0,05)$.

\section{Discusión}

El presente estudio muestra que al menos $40 \%$ de los niños en tratamiento por cáncer que se atienden en el sector público de salud en nuestro medio son seronegativos para VVZ, condición más acentuada en los niños bajo los 10 años, edades en que se concentra el mayor número de pacientes pediátricos en tratamiento por una enfermedad neoplásica y en que la primoinfección por VVZ es más prevalente $^{1}$. La seropositividad de IgG anti VVZ en nuestros pacientes es menor que la comunicada en sujetos sanos en Chile $(61 \%)^{8}$ y en otros países latinoamericanos, como Uruguay $(72 \%)^{10}$ y Brasil $(86 \%)^{7}$, en este mismo grupo etario, lo que podría explicarse porque los niños con cáncer durante sus prolongados tratamientos se encuentran relativamente aislados y no acuden a sus colegios de origen, lo que disminuiría el riesgo de infección.

La inmunidad frente a la infección por VVZ está mediada tanto por actividad humoral como celular; ambas vertientes inmunológicas participan en el control y prevención de la infección, de una manera no del todo comprendida $^{11}$

La primoinfección por VVZ induce una respuesta humoral específica en base a IgM, IgG e IgA séricas e IgA secretoria; de las tres, sólo la IgG específica perdura en el tiempo, aunque en títulos decrecientes, siendo utilizada como marcador de experiencia inmune con el VVZ. La síntesis de anticuerpos neutralizantes anti-varicela del tipo IgG juega un papel protector en los momentos iniciales de la primera fase virémica de la infección ${ }^{12}$, hecho que queda en evidencia con el beneficio obtenido al administrar tempranamente durante la incubación de la enfermedad anticuerpos anti varicela, en diferentes preparados farmacéuticos, en un individuo seronegativo. ${ }^{13}$ De allí se desprende que una persona que posee anticuerpos del tipo IgG anti-VVZ estará protegido contra la primoinfección y tal vez también contra la reinfección.

El estándar de oro para medir protección contra infecciones virales sistémicas es la cuantificación de anticuerpos neutralizantes, técnica lenta, difícil de estandarizar y, en consecuencia, escasamente utilizada con fines clínicos. En el caso de la infección por VVZ, esta cuantificación ha sido reemplazada por la técnica de anticuerpos fluorescentes contra antígeno de membrana (sigla en inglés FAMA) con adecuada correlación hacia inmunoprotección ${ }^{14,15} \mathrm{y}$ posteriormente fue introducida sucesivamente la medición de anticuerpos por técnicas de ELISA contra antígenos virales sin lograr una sensibilidad óptima ${ }^{16}$.

En los últimos años se perfeccionaron los tests de ELISA al utilizar como antígeno glicoproteínas de la envoltura viral purificadas alcanzando una mayor sensibilidad (93-95\%) y preservando su especificidad (cercana a $100 \%)^{17}$; la empleada en este estudio, Ridascreen ${ }^{\circledR}$ VVZ IgG ELISA, ha demostrado tener la mayor correlación con la técnica de FAMA $^{18,19}$ dándole una adecuada solidez a nuestros hallazgos.

La aparición de diversas técnicas y procedencias de tests serológicos para estos efectos, ha planteado a los expertos la duda acerca de cuál es el valor de corte de IgG antiVVZ que correlaciona con protección contra una nueva infección; de los ensayos con vacuna anti-varicela se ha concluido que probablemente un título de ELISA $\geq 5$ unidades es indicativo de protección ${ }^{13}$. Personas con títulos bajo el señalado podrían reinfectarse ${ }^{20}$.

La inmunidad celular, a su vez, participa en varias fases de la infección: el control, en el interior de los macrófagos, de la diseminación hematógena del virus, y el frene de la infección tisular propiamente tal. Se le responsabiliza, además, de mantener en estado latente el genoma del VVZ una vez superada la primoinfección; la ausencia de inmunidad mediada por LTCD4 y LTCD8 y su deterioro representan factores determinante en el desarrollo una varicela grave y de una reactivación del VVZ latente (herpes zoster), respectivamente ${ }^{11}$. Este segundo cuadro suele verse en pacientes inmunocomprometidos seropositivos.

En pacientes inmunosuprimidos como los presentados en el presente estudio, en quienes además la inmunidad celular está globalmente disminuida, no está claramente establecido si los títulos de ELISA $\geq 5 \mathrm{U}$ son protectores como se ha comprobado en personas inmunocompetentes ${ }^{13}$. Así considerado, quizás el porcentaje de pacientes oncológicos atendidos en el seno del PINDA susceptibles a contraer la varicela sea aún mayor que el $40 \%$ observado en este estudio.

Nuestros resultados avalan la necesidad de tomar medidas preventivas que eviten la infección por VVZ en esta población de niños inmunocomprometidos con riesgo de desarrollar una enfermedad grave y eventualmente fatal; lo que además puede afectar el cumplimiento de los protocolos de tratamiento oncológico, situación que podría disminuir las posibilidades de sobrevida del paciente ${ }^{20}$.

La medida más efectiva para evitar una enfermedad grave por VVZ en esta población es la vacunación universal para VVZ a partir del año de vida. En Chile, esta vacuna no está incorporada en el Programa Nacional de Inmunizaciones (PNI).

En niños no vacunados y que no han cursado la en- 
fermedad, las medidas preventivas recomendadas son el uso oportuno de inmunoglobulina específica anti-varicela $\left(\text { VariZIG }{ }^{\circledR}\right)^{21}$, la que debe administrarse precozmente (antes de las $96 \mathrm{~h}$ ) a los pacientes inmunocomprometidos que han sido contactos de pacientes infectados por $\mathrm{VVZ}^{22}$. Esta estrategia ha demostrado ser efectiva en prevenir la enfermedad grave por $\mathrm{VVZ}^{13}$ aunque no es infalible ${ }^{16,23}$.

Debido a la dificultad en adquirir inmunoglobulina específica anti-varicela en forma expedita en nuestro país, se han utilizado otras estrategias preventivas como el uso de aciclovir oral, a partir del día 8 post contacto en dosis de $80 \mathrm{mg} / \mathrm{kg} /$ día, dividida en cuatro tomas, durante 7 días $^{24}$.

La vacuna anti-VVZ no se recomienda de rutina en pacientes inmunocomprometidos, por ser elaborada con virus vivo atenuado; sin embargo, puede ser utilizada en niños con leucemia linfoblástica aguda (LLA) si cumplen con los siguientes requisitos: remisión hematológica de la enfermedad de al menos 12 meses, recuento de linfocitos sobre 1.200 céls $/ \mathrm{mm}^{3}$, de plaquetas mayor a 100.000 céls $/ \mathrm{mm}^{3}$, que no estén recibiendo radioterapia y que la quimioterapia de mantención sea suspendida durante una semana antes y una semana después de la vacunación ${ }^{13,21,22,25-27}$. Esta medida aparece como poco práctica y algo extemporánea, porque sólo es posible de utilizar cuando los pacientes están en una etapa de menor riesgo. Como ya se dijo, la mejor estrategia es la vacunación universal al año de vida incorporada al PNI. Mientras esto no suceda, nuestra recomendación es vacunar a los contactos intradomiciliarios susceptibles al momento del diagnóstico del cáncer y a los pacientes al terminar su tratamiento, una vez que se encuentren inmuno-reconstituidos, de manera de evitar la infección en caso de recaída de la enfermedad oncológica (riesgo aproximado de $30 \%)^{22}$. Por último, para prevenir esta infección en el ambiente intrahospitalario, es fundamental contar con las condiciones que permitan el aislamiento efectivo de los pacientes cursando esta enfermedad ${ }^{20}$.

Limitaciones de este estudio son: la falta de los antecedentes clínicos y de vacunación anti-varicela de los pacientes estudiados y de un grupo control pareado por edad y sexo. Sin embargo, se debe considerar que se trata de pacientes pertenecientes al Sistema Público de Salud, quienes tienen un acceso limitado a la vacuna antivaricela por su costo.

En conclusión, este es el primer reporte de seroprevalencia de VVZ en niños con cáncer en nuestro medio y según nuestros resultados, al menos $40 \%$ de los niños en tratamiento por cáncer son susceptibles de infectarse por este agente, riesgo que es mayor mientras menor edad tiene el paciente.

\section{Resumen}

Las infecciones por virus varicela-zoster (VVZ) en niños inmunocomprometidos presentan una alta morbimortalidad. Se desconoce la seroprevalencia de VVZ en niños con cáncer en nuestro medio. Objetivo: Determinar la prevalencia de anticuerpos anti VVZ en niños sometidos a tratamiento por cáncer (quimioterapia o trasplante de precursores hematopoyéticos). Método: Estudio colaborativo, multicéntrico. Se recolectaron muestras de suero de 281 niños con cáncer y episodios de neutropenia febril (NF) en seis hospitales de Santiago, entre junio 2004 y agosto $2006 \mathrm{y}$ almacenadas a $-70^{\circ} \mathrm{C}^{\circ}$. Doscientas muestras fueron seleccionadas al azar para determinación de $\operatorname{IgG}$ anti VVZ. Resultados: De las 200 muestras de suero obtenidas se excluyeron 21: 12 por ser muestras de un mismo paciente en diferentes episodios de NF y 9 por falta de información. Se analizaron las muestras de 179 niños, $65 \%$ de sexo masculino. Noventa y ocho resultaron positivos (55\%), 72 negativos $(40 \%)$ y 9 indeterminados (5\%). Estratificado por edad: $1-4$ años (32\%), 5-9 años (42\%), 10-14 años (78\%) y mayores de 15 años (88\%). Conclusión: $40 \%$ de los niños en tratamiento por cáncer son seronegativos para VVZ, condición que disminuye en pacientes con mayor edad. Estos resultados apoyan la adopción de medidas que eviten la infección en esta población de niños con riesgo de desarrollar una enfermedad grave y eventualmente fatal.

\section{Referencias bibliográficas}

1.- Heininger U, Seward J F. Varicella. Lancet 2006; 368: 1365 -76.

2.- Folatre I, Zolezzi P, Schimidt D, Marín F, Täger M. Infecciones por virus varicela zoster en menores de 15 años con cáncer. Rev Med Chile 2003; 131:759-64.

3.- Feldman S, Hughes W T, Daniel C B. Varicella in children with cancer. Seventy-seven cases. Pediatrics 1975; 56: 388-97.

4.- Rowland P, Wald E R, Mirro J R Jr, Yunis E, Albo V C, Wollman M R, et al. Progressive varicella presenting with pain and minimal skin involvement in children with acute lymphoblastic leukemia. J Clin Oncol 1995; 13: 1697-703.

5.- Pawlik K M. Varicella zoster infection in the immunocompromised child. J Soc Pediatr Nurs. 1998; 3: 13-20.

6.- Centers for Disease Control and Prevention (CDC). Prevention of varicella: recommendations of the Advisory Committee on Immunization Practices (ACIP). MMWR Recomm Rep 2007; 56(RR-4): 1-40.

7.- Clemens S, Azevedo T, Fonseca J, Cavalcante Silva A, Da Silveira T, Clemens R. Seroepidemiología de la varicela en Brasil:
Resultados de un estudio prospectivo transversal. Rev Chil Pediatr 2001; 72: 538-47.

8.- Lagos R, Potin M. Tanaka J. Seroprevalencia de anticuerpos antivaricela zoster en población sana del área norte de Santiago. Libro de resumen. XIV Congreso Chileno de Infectología, Valdivia, 1997.

9.- Becker A. Pronóstico de vida y secuelas del tratamiento del cáncer en los niños. Rev Chil Pediatr 2003; 74: 520-23.

10.- Quian J, Rüttimann R, Matrai L. Prevalencia de anticuerpos contra varicela en una población de Montevideo, Uruguay. Arch Pediatr Urug 2004; 75: 133-8. 
11.- Gershon AA, Silverstein SJ. Varicella-Zoster Virus. En: Clinical Virology. DD. Richman, RJ Whitley, FG Hayden, eds. Thirth Edition. 2009. ASM Press, Washington DC. pp 451-73.

12.- Fisher J P, Bate J, Hambleton S. Preventing varicella in children with malignancies: what is the evidence? Curr Opin Infect Dis 2011; 24: 203-11.

13.- Gershon A A, Takahashi M, Seward J F. Chapter 35: Varicella vaccine. En Vaccines. Plotkin S, Orenstein W, Offit P, eds. fifth edition 2008. Saunders Elsevier, pp: 915-58.

14.- Williams V, Gershon A, Brunell P A. Serologic response to varicela-zoster membrane antigens measured by direct immunofluorescence. J Infect Dis 1974; 130: 669-72.

15.- Li S, Chan I S, Matthews H, Heyse J F, Chan C Y, Kuter B J, et al. Inverse relationship between six week postvaccination varicella antibody response to vaccine and likelihood of long term breakthrough infection. Pediatr Infect Dis J 2002; 21 (4): 337-42.

16.- Arvin A M. Chapter 205: Varicella-Zoster Virus. En: Principles and Practice of Pediatric
Infectious Diseases. Long SS., Pickering LK., Prober CH G., fourth edition 2012. Elsevier Saunders. pp: 1035-44.

17.- Breuer J, Schmid D S, Gershon A A. Use and limitations of varicella-zoster virus-specific serological testing to evaluate breakthrough disease in vaccinees and to screen for susceptibility to varicella. J Infect Dis 2008; 197 Suppl 2: S147-51.

18.- Sauerbrei A, Wutzler P. Serological detection of specific IgG to varicella-zoster virus by novel ELISA based on viral glycoprotein antigen. Clin Lab 2009; 55 (1-2): 1-7.

19.- Sauerbrei A, Wutzler P. Serological detection of varicella-zoster virus-specific immunoglobulin $\mathrm{G}$ by an Enzyme-Linked Immunosorbent Assay using glycoprotein antigen. J Clin Microbiol 2006; 44: 3094-7.

20.- Manley S, Mallinson H, Caswell M, Keenan R, Pizer B. Chickenpox in varicella IgG positive patients: experience of a regional paediatric oncology centre. Pediatr Blood Cancer 2008; 51: $540-2$

21.- American Academy of Pediatrics. VaricellaZoster Infections. In: Pickering LK, Backer CJ,
Kimberlin DW, Long SS, eds. Red Book: 2012 Report of the Committee on Infectious Disease. Elk Grove Village, IL: American Academy of Pediatrics; 2012: 774-89.

22.- Leung A, Kellner J, Davies D. Chickenpox: An update. J Pediatr Infect Dis 2009;4: 343-50

23.- Adler A L, Casper C, Boeckh M, Heath J, Zerr D M. An outbreak of varicella with likely breakthrough disease in a population of pediatric cancer patients. Infect Control Hosp Epidemiol 2008; 29: 866-70.

24.- Cofré J. Varicela: Consultas frecuentes acerca de su tratamiento y el manejo de los contactos. Rev Chilena Infectol 2008; 25: 390-94.

25.- Royal College of Paediatrics and Child Health. Immunisation of the immunocompromised child. Best Practice Statement. London: RCPCH, 2002.

26.- Sartori A M. A review of the varicella vaccine immunocompromised individuals. Int J Infect Dis 2004; 8: 259-70.

27.- Sierrasesumaga A L. Complicaciones de la varicela en el paciente oncológico y en el transplante de órganos. An Pediatr (Barc) 2003; 59 (Supl 1): 32-40. 